\title{
CRITERIOS DE SELECCION Y EXPECTATIVAS DE CONCLUSION DE CARRERA EN LA UNIVERSIDAD DE COSTA RICA
}

\author{
Irene Méndez Vega
}

\section{Introducción}

La propuesta de reforma curricular denominada "Estructura de Ciclos Básicos por Area", dio inicio en la Universidad de Costa Rica, en 1985, con el establecimiento de los ciclos básicos de Biociencias e Ingenierías, a manera de plan piloto, según acuerdo del Consejo Universitario (U.C.R., Consejo Universitario, 1984).

La "reforma curricular" fue considerada como una de las actividades prioritarias de la Institución, a ejecutar de 1985 a 1990, según consta en el "Plan Quinquenal de Desarrollo Institucional" de la Universidad, para dicho periodo (U.C.R., Rectoría, 1985; III-39).

Su fundamentación teórica, así como la formulación de sus objetivos y de las recomendaciones para su ejecución, aparecen señalados en el documento de la Rectoría "Estructura de los Ciclos Básicos por Area. Propuesta de Reforma Curricular", publicado en 1984 (U.C.R., Rectoría, 1984).

En dicho documento se enuncian dos objetivos que le ofrecen al estudiante universitario la opción de elegir entre varias carreras del área escogida, sin pérdida de tiempo para él ni de recursos para la Institución; uno es que pueda optar por otra u otras carreras si la opción inicialmente elegida no le satisface; y el otro, es brindarle una orientación para que pueda desenvolverse con seguridad en el medio universitario, descubrir sus propias aptitudes, y elegir acertadamente su campo profesional (U.C.R., Rectoría, 1984; 16).

Entre las recomendaciones de la propuesta en relación con estos objetivos, está la de establecer como política de admisión a las carreras, que el estudiante ingrese solo des- pués de que haya aprobado el Ciclo Básico (U.C.R., Rectoria, 1984; 17).

De acuerdo con las metas fijadas en el Plan Quinquenal de Desarrollo Institucional (U.C.R., Rectoría, 1985), se pretende que, posterior a la evaluación del Ciclo Básico de Biociencias, la estructura de ciclos básicos se extienda a otras áreas del conocimiento, y se hace referencia, expresamente, a las de Ciencias Sociales y Artes y Letras (U.C.R., Rectoría, 1985; V-35).

Para apoyar al Ciclo Básico de Biociencias se estableció en la Universidad, el "Programa de Recursos Didácticos para el estudio de las Carreras" (PREDEC), con el objetivo de orientar, coordinar, diagnosticar, y ayudar al estudiante en el escogimiento de su carrera (U.C.R., OPLAU, 1985; 26).

El proposito de este artículo, es presentar los resultados que muestran la opinión estudiantil en relación con los factores que, a su juicio, incidieron realmente en la escogencia de su carrera profesional, y, conocer sus expectativas en cuanto al número de años para concluirla.

Los sujetos de estudio, son parte del grupo de estudiantes que se vieron afectados por la puesta en marcha de la propuesta de reforma curricular, quienes, para optar por alguna carrera del Area de Biociencias, debieron inscribirse a partir de 1985 , en el ciclo básico respectivo.

\section{Los asuntos estudiantiles en la investigación universitaria}

Los estudios universitarios constituyen de por si, un reto importante para los estu- 
diantes, en el que intervienen una amplia gama de factores socioeconómicos, psicosociales y estructurales, que determinan la condición académica estudiantil.

Desde inicios de los años sesenta, la Universidad de Costa Rica se ha interesado por conocer, sistemáticamente, los factores asociados al rendimiento estudiantil, como un medio para analizar la respuesta del estudiante a las disposiciones académicas de la Institución. En esta tarea, la Universidad contó con la labor pionera realizada por la Facultad de Ciencias y Letras, la que fue continuada por el Instituto de Investigaciones Psicologicas (IIP), $y$, recientemente, además, por la Oficina de Planificación Universitaria (OPLAU), el Instituto de Investigaciones para el Mejoramiento de la Educación Costarricense (IIMEC), el Departamento de Docencia Universitaria (DEDUN), la Oficina de Salud, y algunas unidades académicas.

Este trabajo ha sido apoyado también, externamente, por los estudios que efectúa la Oficina de Planificación de la Educación Superior (OPES), la cual, con cierta regularidad, publica estudios sobre la situación de la población estudiantil graduada en las universidades estatales, regidas por el Consejo Nacional de Rectores (CONARE).

Dentro de los temas que han sido objeto de estudio en la Universidad de Costa Rica en materia estudiantil, se encuentran los orientados a conocer la relacion entre los estudiantes y horas de estudio (Adis, Azofeifa y Umaña, 1961), que determino el tiempo real ocupado por los estudiantes para el estudio; los que proponen conocer los problemas que plantea el ingreso a la Universidad (Adis y Azofeifa, 1962), que estudian las inquietudes que sufre la población estudiantil interesada en seguir estudios universitarios, al tener que enfrentarse a una prueba de admisión.

Otro estudio (Chaverri, 1969) vers6 específicamente sobre el rendimiento estudiantil, y destaca que el rendimiento insatisfactorio que presenta el estudiante universitario, se manifiesta desde su primer año de ingreso a la Universidad, durante el cual, escasamente, cumple con la mitad de la carga académica que establecen las distintas carreras para cada ciclo lectivo, lo que redundará en un alargamiento de la carrera (Chaverri, 1969; 201).
También hay estudios que relacionan el rendimiento académico del estudiante, por tanto, su éxito o fracaso en carrera, con el promedio obtenido en la prueba de admision a la Universidad (De León-Páez y Wong de, 1973 y 1990). Estos estudios comparan los resultados obtenidos por los estudiantes en su primer año de estudios, con la nota de admision, en donde se concluye que hay una relación directa entre rendimiento académico y puntaje de admisión ( De León-Paez, Wong de M., 1973; 159).

En la década de los setenta, el interés institucional por los estudios sobre el rendimiento académico estudiantil, se ve fortalecido por la disposición del Consejo Universitario, de crear, en 1971, el "Programa de Rendimiento Académico", bajo la responsabilidad del Instituto de Investigaciones Psicologicas (U.C.R., Consejo Universitario, 1971).

La tarea encomendada fue la de encontrar los factores asociados al éxito o fracaso académico de los estudiantes universitarios. En atención a esta demanda, se conceptualizaron, diseñaron y desarrollaron proyectos de investigación, que permitieron empezar a configurar un perfil de algunos fenomenos y características que participan en el proceso enseñanza-aprendizaje, operante en la Universidad de Costa Rica.

Dentro de este Programa, destacan los estudios sobre rendimiento académico por escuelas y departamentos en la Institución (Beirute, González, Otero, 1972), que pretendieron reunir información acerca de la matrícula, promoción y deserción en la Universidad; los que estudian los factores sociodemográficos, carga académica y rendimiento académico (Otero, 1976), y los que investigan cuáles son las relacionés entre los factores sociodemográficos y académicos con la valoración que tiene el estudiante de sí mismo (Brenes y Raven, 1977).

Otro tema de interés fue conocer el nivel de aspiraciones que tienen los estudiantes universitarios, con base en el rendimiento académico que han obtenido en la Universidad de Costa Rica (Otero, Raven, Alvarez, 1977 ). Institucionalmente, este tipo de análisis ha sido de gran importancia, ya que les ha permitido a las autoridades universitarias plantearse la problemática psico-social del estu- 
diante universitario, y ha dejado clara la necesidad de profundizar en los factores que inciden en el rendimiento académico más allá de la nota, simplemente.

En los años ochenta, los temas que preocupan a los investigadores $y$, fundamentalmente, a las autoridades universitarias por las implicaciones presupuestarias, son los indices de deserción en términos de estudiante-curso, y los factores asociados a su prevalencia.

Otero (1983), estudió la forma de operacionalizar, describir y analizar los indicadores de deserción de la Universidad de Costa Rica, así como la de identificar, describir y analizar la relación que existe entre los componentes de la deserción y el área de estudio de preferencia para los estudiantes universitarios. También se preocupó por establecer, describir y analizar el tipo de relación que prevalece entre los indicadores de deserción y las características sociodemograficas de los estudiantes universitarios, según el área de estudios. Entre los indicadores de deserción que mostró el estudio, están el retiro justificado, la no presentación a examen final, la pérdida del curso por falta de prácticas, y la interrupción de estudios. En cuanto a la relación entre los indicadores de deserción y el área de estudio, se estableció que, tanto en Artes y Letras como en Ciencias Sociales, adquieren mayor magnitud que en Ciencias de la Salud (Otero, 1983; 15).

Con la creación en la Universidad de Costa Rica de la Oficina de Planificación Universitaria (OPLAU) en 1984, se enfocan, cuantitativamente, los problemas relacionados con el rendimiento estudiantil. Se analizan diferentes variables tales como distribucion de las cargas de estudio, el aprovechamiento, la deserción, la repetición de cursos, y la simulación del efecto del "Reglamento de Régimen Académico Estudiantil" sobre el estudiante, que aportan datos actualizados sobre el perfil estudiantil.

A pesar de que la Universidad de Costa Rica ha abordado la problemática estudiantil considerando aspectos académicos y socioeconómicos, una vez que se produce el ingreso a la Institución, quedan temas que necesitan mayor profundización, entre ellos conocer cuál es el comportamiento estudiantil en relación con la escogencia de carrera, y cuál es la incidencia de esta variable en el éxito o fraca- so del estudiante, para concluir una carrera universitaria. De igual manera, es importante detectar y atender las causas que inciden en la duración de los estudios.

Entre las investigaciones que se refieren a estos temas, se pueden mencionar las tendientes al análisis de los factores asociados con la escogencia de carrera universitaria de parte del estudiante al ingresar a la Universidad, y su perseverancia en dicha escogencia (Thomas, 1970), así como los que estudian las aspiraciones de ingreso a la Universidad, de parte de estudiantes de provincia (Thomas, 1971).

El primer estudio se efectúo por solicitud de la Comisión Coordinadora del Programa de Formación de Profesores en Enseñanza Media, en la Universidad de Costa Rica. Los resultados obtenidos sugieren que, tanto antes de ingresar a la Universidad, como después de un año de permanencia en la Institución, son pocos los estudiantes que se han definido respecto a la carrera que piensan seguir. Asimismo, que los cambios de interés observados en cuanto a la escogencia estudiantil hacia una u otra carrera, no pueden incriminarse a problemas de índole académica, pues no se encontraron diferencias significativas entre el rendimiento académico obtenido por los estudiantes que optaron por el profesorado en Enseñanza Media u otras carreras universitarias, luego de su primer año de estudios universitarios (Thomas, 1970; 8).

Sobre las causas que inciden en la duración de los estudios, una investigación realizada por la Oficina de Planificación de la Educación Superior (CONARE, OPES, 1989), sobre la situación laboral y otras características de los graduados de las cuatro universidades estatales, determinó que, -si bien los planes de estudio de las diferentes carreras ofrecidas por las universidades estatales establecen una duración de 2, 4 y 5 años para obtener los grados de diplomado, bachillerato y licenciatura, respectivamente, un alto porcentaje de los graduados tardó un período de aproximadamente el doble del tiempo esperado. Por otro lado, un porcentaje significativo de los graduados cursó estudios en una carrera distinta a la que había elegido inicialmente. Muchos de los graduados atribuyeron este hecho a que, al ingresar a la universidad, no 
tenían suficiente conocimiento para la elección de carrera (CONARE, OPES, 1989; 14).

En relación con la Universidad de Costa Rica, el estudio de OPES establece que el tiempo de permanencia, entendido como el transcurrido desde que el estudiante ingresa a la Universidad, hasta que obtiene el título, es en promedio de 7,6 años para el diplomado, 8,6 para el bachillerato y 11,1 para la licenciatura; promedios superiores inclusive a los registrados por la Universidad Estatal a Distancia (CONARE, OPES, 1989; 45).

En cuanto al tiempo de graduación, calculado a partir del momento en que el estudiante ingresa a carrera, hasta la fecha en que se 'gradúa, este es en la Universidad de Costa Rica de 4,8 años para el diplomado, 6,8 para el bachillerato y 9,2 para la licenciatura (CONARE, OPES, 1989; 46).

Del total de graduados en 1986, la Universidad de Costa Rica ocupo el porcentaje más alto en cuanto a incidencia de graduados que cambiaron una o varias veces de carrera. Este corresponde a un $23,7 \%$, mientras que en el Instituto Tecnologico de Costa Rica fue de un $6,2 \%$, en la Universidad Nacional de un $9,3 \%$ y en la Universidad Estatal a Distancia, de un 5,1\% (CONARE, OPES, 1989; 47).

Con base en los resultados obtenidos y dada la prolongada permanencia de los estudiantes en las universidades estatales, OPES realiz6 un estudio en 1990 para determinar el efecto directo e indirecto que tienen sobre el período de permanencia, variables como sexo, edad en el momento de ingresar a la universidad, condición laboral durante el tiempo de estudio, cambio de carrera, otro diploma obtenido en diferente carrera, interrupción de estudios, período transcurrido entre el egreso y la graduación. También se propuso establecer si existen diferencias significativas entre los factores que afectan la permanencia de los estudiantes en las distintas universidades estatales (CONARE, OPES, 1990).

Entre los principales resultados de este estudio, se determinó que, si bien los factores que afectan la permanencia de los estudiantes varían según la institución donde hayan realizado los estudios y el grado académico obtenido, hay algunos que son comunes a las cuatro instituciones estatales; entre ellos, la interupción de estudios, la cual aparece como la variable que más afecta, a excepción de las licenciaturas en la Universidad Nacional. El estado civil es otra de las variables que más afectan, aunque no en la Universidad Estatal a Distancia. Otro factor es el tiempo que transcurre entre el momento en que los estudiantes concluyen las materias del plan de estudio y la fecha en que obtienen su diploma de licenciados, lo cual podría deberse al tiempo que tardan para hacer los trabajos finales de graduación. En menor grado, el estudio de OPES establece que factores de caracter administrativo-institucional, tales como horarios y recursos disponibles, influyen en la prolongada permanencia estudiantil en carrera (CONARE, OPES, 1990; 12).

\section{Metodologia}

Con base en los archivos de matrícula de 1985, y en los padrones por escuela para 1991, aportados por la Oficina de Registro de la Universidad de Costa Rica, se procedio a determinar cuáles estudiantes, con carné 85 , se habían empadronado en el primer ciclo lectivo de 1985 en "Biociencias", no habian tenido interrupciones en sus estudios universitarios, y estaban registrados, en 1991, como estudiantes regulares, en los padrones de las siguientes carreras:

\section{Bachillerato en Ciencias Médicas y Licenciatura en Medicina y Cirugía; \\ Licenciatura en Microbiología y Química Clínica; \\ Licenciatura en Farmacia; \\ Licenciatura en Zootecnia; \\ Bachillerato y Licenciatura en Fitotecnia, y, \\ Licenciatura en Tecnōogía de Alimentos.}

Estas carreras se seleccionaron, con base en la resolución VD-1875-84, emitida por la Vicerrectoría de Docencia en noviembre de 1984, la cual establecio, precisamente, que a partir de 1985, el requisito de ingreso a esas y otras carreras, se basaría en el rendimiento académico del estudiante, en relación con los cursos del ciclo básico de materias comunes (química, matemática, biología y física).

Este procedimiento dio como resultado una población de 168 sujetos, cuya distribu- 
cion por carrera es la siguiente: 50 en Medicina, 24 en Microbiología, 38 en Farmacia, 28 en Odontología, 9 en Tecnología de Alimentos, 6 en Zootecnia y 13 en Fitotecnia.

Una vez definida la población, se procedio a ubicarla por cursos o actividades, dentro y fuera de la Sede Rodrigo Facio, para pasarles un cuestionario y obtener información sobre los siguientes aspectos:

1. Momento en que tomo la decisión sobre qué carrera estudiar.

2. Criterios utilizados en la escogencia de - carrera.

3. Grado universitario más alto al que aspira y ciclo lectivo en que espera terminar la carrera.

Esta tarea se realizo durante los meses de abril y mayo de 1991, para lo cual se contó con la colaboración de las Unidades Académicas involucradas.

El porcentaje de estudiantes en niveles avanzados de carrera fue relativamente alto $(82,1 \%)$ por lo que hubo que visitarlos fuera de la Sede Rodrigo Facio (hospitales del país, empresas, estaciones experimentales, etc.), en donde cumplian con las prácticas especificadas dentro de los planes de estudio, para los últimos años de carrera (Méndez, 1991; 147-149).

El cuestionario fue sometido a juicio de expertos, se codifico previamente, y para el procesamiento de los datos se utilizó el paquete estadistico "SPSS/PC".

El promedio de respuesta fue del $83,33 \%$, lográndose el $100 \%$ en las carreras de Zootecnia, Tecnología de Alimentos y Microbiologia, un $92,1 \%$ en Farmacia, $84,61 \%$ en Fitotecnia, $75 \%$ en Odontología y $70 \%$ en Medicina, para un total de 140 estudiantes.

De esta forma, la muestra total estuvo conformada por valores porcentuales del $70 \mathrm{al}$ $100 \%$ del total de los estudiantes considerados para cada una de las carreras.

\section{Resultados}

Los resultados se presentan por medio de cuadros generales, para cada uno de los aspectos analizados.
El cuadro 1 resume la información sobre el momento en que los estudiantes toman la decisión de escoger su carrera. Se observa que el $71 \%$ de los estudiantes eligieron su carrera desde el momento en que se encontraban en el colegio, y el $23 \%$ se decidio en la Universidad.

Cuadro 1

Estudiantes de la Universidad de Costa Rica, carne 85 , según momento en que decidieron su carrera profesional. 1991

\begin{tabular}{|c|c|c|c|}
\hline \multicolumn{2}{|r|}{ Momentos } & Frecuencia & $\%$ \\
\hline 1 & $\begin{array}{l}\text { Se decidio desde que estaba en } \\
\text { el colegio y la manruvo aún } \\
\text { despues de ingresar a la Universidad }\end{array}$ & 100 & 71,4 \\
\hline 2. & $\begin{array}{l}\text { Tomó la decision en el Colegio } \\
\text { pero la varió en la Universidad. }\end{array}$ & 13 & 9,3 \\
\hline 3. & $\begin{array}{l}\text { No tuvo decisión previa a su ingreso } \\
\text { a la Universidad, sino que la tomó } \\
\text { mientras cursaba el Ciclo Básico de } \\
\text { Biociencias. }\end{array}$ & 19 & 13,6 \\
\hline 4 & $\begin{array}{l}\text { No pudo decidirse mientras cursaba } \\
\text { el Ciclo Bksico de Bio-ciencias, sino } \\
\text { que opto por matricularse en varias } \\
\text { carreras para decidirse despues. }\end{array}$ & s & 3,6 \\
\hline 5. & $\begin{array}{l}\text { Aunque ya está en carrera, } \\
\text { todavia considera que es mejor } \\
\text { cambiarse a otra. }\end{array}$ & 3 & 2,1 \\
\hline To & & 140 & 100,00 \\
\hline
\end{tabular}

Estos resultados indican que, en opinión de un alto porcentaje de estudiantes, el Ciclo Básico de Biociencias no representa para el estudiante una opción importante en la elección de su carrera. Sin embargo, es obvio que más de una quinta parte de los estudiantes que ingresa a la Universidad, como los de la muestra, se deciden por una carrera específica o rectifican la decisión que traían al ingresar, en el transcurso de los primeros años de vida universitaria.

En relación con los criterios que influyeron sobre el estudiante para la escogencia de su carrera, se observa en el cuadro 2 , que la decisión personal basada en la convicción de que la carrera respondía a sus aptitudes y 
capacidades, prevaleció sobre los otros criterios, en el $73,6 \%$ de la población estudiantil.

Cuadro 2

Estudiantes de la Universidad de Costa Rica, carne 85, según criterio utilizado para escoger carrem. 1991

\begin{tabular}{lcc}
\hline \multicolumn{1}{c}{ Criterio } & Frecuencia & $\%$ \\
\hline 1. La familia influyo fuerternente & 10 & 7,1 \\
$\begin{array}{l}\text { 2. De acuerdo con su criterio y } \\
\text { aspiraciones, era la carrera que } \\
\text { podia ofrecerle buena posición } \\
\text { economica y social }\end{array}$ & 21 & 15,0 \\
$\begin{array}{l}\text { 3. Consideró que esa carrera era } \\
\text { la que respondia a sus aptitudes } \\
\text { y capacidades }\end{array}$ & 103 & 73,6 \\
$\begin{array}{l}\text { 4. No sabe todavía por que escogio } \\
\text { la carrera }\end{array}$ & 6 & 4,3 \\
\hline \begin{tabular}{l} 
Total \\
\hline
\end{tabular} & 140 & 100,0 \\
\hline
\end{tabular}

La familia influyó en el $7,1 \%$ de los estudiantes y, la expectativa personal sobre la base de una mejor posición económica y social, determinó la escogencia en el $15 \%$ de los estudiantes. Un porcentaje muy bajo no sabía aún, luego de siete años de permanencia en la Universidad, por qué escogió la carrera que sigue.

En relación con el grado universitario más alto al que aspiran los estudiantes, se observa en el cuadro 3, que el $100 \%$ se manifestó por la licenciatura y de ellos, el $41,4 \%$ expresó su deseo de continuar con estudios a nivel de posgrado.

Cuadro 3

Estudiantes de la Universidad de Costa Rica, carne 85 , según grado universitario al que aspiran. 1991

\begin{tabular}{lcr}
\hline Grado Universitario & Frecuencia & $\%$ \\
\hline Bachillerato & 0 & 0,0 \\
Licenciatura & 140 & 100,0 \\
$\begin{array}{l}\text { Especialidad/posgrado } \\
\text { después de la licenciatura }\end{array}$ & 58 & 41,4 \\
\hline Total & 140 & 100,0 \\
\hline
\end{tabular}

En cuanto a las expectativas sobre la duración de los estudios que tiene esta población estudiantil, el cuadro 4 muestra que el $97,9 \%$ aspira concluir su carrera entre el primer ciclo lectivo de 1991 y el primero de
1992, y entre el segundo de 1992 y el primero de 1993 , a razón de un $36,4 \%$ y un $61,5 \%$ respectivamente, lo que implicaría para el primer grupo una duración de 6,5 a 7,5 años lectivos, y de 8 a 8,5 para el segundo.

Cuadro 4

Estudiantes de la Universidad de Costa Rica, came 85, según tiempo estimado para concluir estudios. 1991

\begin{tabular}{lcccc}
\hline $\begin{array}{c}\text { Ciclo } \\
\text { lectivo/Ano }\end{array}$ & $\begin{array}{c}\text { Total/Años } \\
\text { lectivos }\end{array}$ & Frecuencia & $\%$ \\
\hline I & 1991 & 6,5 & 18 & 12,8 \\
II & 1991 & 7,0 & 14 & 10,0 \\
I $\quad 1992$ & 7,5 & 19 & 13,6 \\
II $\quad 1992$ & 8,0 & 47 & 33,6 \\
I $\quad 1993$ & 8,5 & 39 & 27,9 \\
II & $19930+$ & $9,00+$ & 3 & 2,1 \\
\hline Total & & 140 & 100,0 \\
\hline
\end{tabular}

Un pequeño porcentaje de estudiantes, $2,1 \%$, espera concluir carrera, luego de más de nueve años de estudios universitarios.

\section{Discusión y conclusiones}

La Universidad de Costa Rica dedica parte de sus esfuerzos a la investigación cualitatita y cuantitativa sobre la población estudiantil, una vez que esta ha ingresado a la Institucion. No obstante, la importancia de retomar como tema de estudio las expectativas que tienen los jóvenes en cuanto a educación superior desde que cursan la secundaria, se refleja en los resultados presentados en este artículo.

De acuerdo con los escasos estudios que hay sobre las variables que influyen en el escogimiento de la carrea profesional, son pocos los estudiantes que se definen tempranamente respecto a qué carrera estudiar. Esta situación se comporta diferente en la población objeto de este estudio, en donde, por lo contrario, un alto porcentaje de estudiantes $(71,4 \%)$, había escogido carrera desde la secundaria y mantuvo esa decisión en la Universidad. 
No se cuenta con información sobre el proceso de orientación vocacional que tuvo el estudiante en su colegio, en relación con la oferta académica de la Universidad de Costa Rica, la que sin duda vendría a aportar mayores criterios para valorar este cambio de actitud.

Otro aspecto importante que se deduce de los resultados, es que el proceso de orientación paralelo al Ciclo Básico de Biociencias, por medio del Programa de Recursos Didácticos para el Estudio de las Carreras (PREDEC), pudo haber influido en la escogencia de carrera de un $23 \%$ de los estudiantes de la muestra, mientras que, en apariencia, no tuvo ningún efecto en el $5,7 \%$ de la población que optó por elegir carrera hasta después de concluir con el Ciclo Básico, o bien que aún se mantiene indecisa.

Esta situación debe ser considerada por las autoridades universitarias, con miras a fortalecer y/o replantear el proceso de orientación profesional al estudiante, en general, desde el momento que cursa los Estudios Generales. Lo anterior sería de gran ayuda tanto para los estudiantes de nuevo ingreso, como para los que no están ubicados en carrera, pese a tener varios años en la Institución.

Otra conclusión a que se llega en este artículo, dificil de cotejar con datos anteriores por falta de los mismos, es que la escogencia de carrera de casi tres cuartas partes de los estudiantes de la muestra, estuvo orientada por un reconocimiento personal, sin aparentes influencias familiares o sociales, en donde el estudiante toma la decisión motivado por la seguridad de que la carrera responde a sus aptitudes y capacidades, lo que le permitirá concluirla exitosamente.

Mientras que la familia influyo en la escogencia de carrera de un $7,1 \%$ de los estudiantes, un $15 \%$ se oriento por la posibilidad de lograr una buena posición economica y social, y finalmente, un $4,3 \%$, porcentaje cercano a los que no habían escogido carrera luego de cursar el Ciclo Básico de Biociencias, manifesta que aún, en 1991, no sabían por qué escogieron la carrera que cursan.

Estos tres últimos casos merecen ser estudiados a fin de darle seguimiento a esos estudiantes, inclusive una vez graduados, para poder inferir si tales situaciones influyen en la calidad del desempeño profesional y actitutd humanista, que predica el Estatuto Organico de la Universidad de Costa Rica. Asimismo, estos datos confirman la necesidad que tiene una buena parte de los estudiantes universitarios de asesoramiento para una mejor definición de su vocación profesional.

Es importante notar que el $100 \%$ de la muestra aspira a obtener el grado académico de licenciatura. Lo anterior probablemente responda a que solo dos carreras, Fitotecnia y Medicina, ofrecen el bachillerato universitario previo a la licenciatura, mientras que en Odontología, Farmacia, Microbiología, Tecnología de Alimentos, ofrecen la licenciatura directamente.

Además, de acuerdo con el mercado laboral, es posible que el bachillerato no ofrezca mayores alternativas de empleo dentro de estos campos profesionales, sino que más bien exija niveles de especialización, hacia los cuales se dirige el $41,4 \%$ de los estudiantes, al manifestarse por el posgrado.

En relación con las expectativas de los estudiantes para concluir su carrera, los resultados obtenidos, si bien no son los 6ptimos, sí son satisfactorios tanto para la Institución como para los mismos estudiantes y la sociedad costarricense.

A finales de 1992, un $70 \%$ pudo haber concluido sus estudios en la carrera elegida, y un $28 \%$ lo hara en el primer ciclo lectivo de 1993. Esto implica que a lo sumo, para la mayoría de los estudiantes, el período lectivo para obtener una licenciatura será igual o inferior a 8 años y medio.

Si se relacionan estos datos con el plazo que para tal fin indican los planes de estudio, el cual es de 10 ciclos ( 5 años), para las licenciaturas en Tecnología de Alimentos, Fitotecnia y Zootecnia; y, 12 ciclos (6 años) para Medicina, Microbiología, Odontología y Farmacia, se desprende que, la diferencia con el tiempo que el estudiante ocupa efectivamente, es, en la mayoría de los casos de uno a tres años.

Esta diferencia no resulta tan marcada en la muestra de estudiantes, si se toma en consideración que el tiempo de permanencia promedio por estudiante, en la Universidad de Costa Rica, es de 11,1 años para obtener la licenciatura. 
A pesar del aparente éxito académico de estos estudiantes, es necesario apoyar teórica y metodológicamente los datos obtenidos, con investigaciones interdisciplinarias que permitan analizar el entorno socioeconómico y cultural del estudiante, antes y después de su ingreso a la Universidad, y relacionarlo con otras variables, tales como tiempo dedicado al estudio, rol del profesor consejero, calidad de los servicios ofrecidos al estudiante por la Institucion, estructura curricular de los planes de estudio, entre otros.

Finalmente se recomienda efectuar estudios como el que aquí se presenta, con estudiantes de toda la Universidad de Costa Rica, para determinar si hay diferencias significativas en cuanto a los dos aspectos analizados: criterios de selección y expectativas de conclusión de carrera, en función de cada una de las áreas de estudio que ofrece la Universidad.

\section{Bibliografia}

Adis, G., Azofeifa, I., Umaña, R. Estudiantes y boras de estudio. Publicaciones de la Universidad de Costa Rica, 1961.

Adis, G., Azofeifa, I. Problemas que plantea el ingreso a la Universidad. U.C.R., Centro de Investigaciones Psicológicas, 1962.

Adis, G., Thomas, P. La Universidad y el Rendimiento Académico. U.C.R., Centro de Investigaciones Psicológicas, 1966.

Beirute, L., González, M., Otero, L. Rendtmiento Académico por escuelas y Departamentos en la Universidad de Costa Rica. U.C.R., Instituto de Investigaciones Psicologicas, 1972.

Brenes, A. y Raven, N. Algunos factores soctodemograficos $y$ académicos y su relación con la valoración de si mismo. U.C.R., Instituto de Investigaciones Psicologicas, 1977.

CONARE. La stiuactón laboral y otras caractenisticas de los graduados de 1986 de las Universidades Estatales. San José: OPES-07/89, 1989.
CONARE. Estudio exploratorio sobre los factores que inciden en el periodo de perm anencia de los estudiantes en las Instituciones de Educacion Superior. San José: OPES-28/90, 1990.

Chaverri, G. "Reglamento académico de los estudiantes de Estudios Generales". Revista de la Universidad de Costa Rica No.27: 201-208, 1969.

De León-Páez, O. y Wong de, M. "El examen de admisión y el rendimiento del estudiante en su primer año académico". Revista de la Universidad de Costa Rica No. 35: 159-164, 1973.

González, M. y De León-Páez, O. "Diferentes variables criterio para validez predictiva de admisión y sus componentes". Revista Educación. Vol. 14: 137-142, 1990.

Méndez, I. "Exito académico de los estudiantes de la Universidad de Costa Rica con carné 85, que ingresaron al Ciclo Básico de Biociencias en 1985 y cursan carreras de Agronomía y Salud". Tesis de Licenciatura en Currículo. Facultad de Educación-Sede de Guanacaste, 1991.

Otero, L. Factores sociodemograficos, carg a académica y rendimiento académico. U.C.R., Instituto de Investigaciones Psicologicas, 1976.

Otero, L., Raven, N. y Alvarez, A. Nivel de aspiraciones $y$ rendimiento académico en la Untversidad de Costa Rica. U.C.R., Instituto de Investigaciones Psicologicas, 1977.

Otero, L. Desercion e indicadores de rendimiento en la Universidad de Costa Rica. U.C.R., Instituto de Investigaciones Psicológicas, 1983.

Thomas, P. Actitud Académica y Desercion. U.C.R., Centro de Investigaciones Psicologicas, 1969. 
Thomas, P. Los estudiantes y la escogencla de carreras untversitarias. U.C.R., Instituto de Investigaciones Psicologicas, 1970.

Thomas, P. Aspiraciones de Ingreso a la Universidad por parte de estudiantes de prouincia. U.C.R., Instituto de Investigaciones Psicologicas, 1971.

Universidad de Costa Rica. Consejo Universitario. Acuerdos sobre rendimiento estudiantil, 1971.

Universidad de Costa Rica. Consejo Univer-

- sitario. Acta Sesión 3140, 8 mayo 1984.

Universidad de Costa Rica. Estatuto Organico. San José: Publicaciones de la Universidad de Costa Rica, 1984.

Universidad de Costa Rica. Oficina de Planificacion Universitaria. Plan de Desarrollo 86-90. La condictón academica estudiantil en la Universidad de Costa Rica. Publicaciones de la Universidad de Costa Rica, 1985.

Universidad de Costa Rica . Oficina de Planificación Universitaria. La reform a curricular en la Universidad de Costa Rica. Publicaciones de la Universidad de Costa Rica, 1985.

Universidad de Costa Rica. Oficina de Planificacion Universitaria. Plan Operativo 1990. 2 tomos. Publicaciones de la Universidad de Costa Rica, 1990.

Universidad de Costa Rica. Rectoría. Estructura de los Ciclos Basicos por Are a Propuesta de Reforma Curricular. Publicaciones de la Universidad de Costa Rica, 1984.

Universidad de Costa Rica. OPLAU. La reforma curricular en la Universidad de Costa Rica. Publicaciones de la Universidad de Costa Rica, 1985.

Universidad de Costa Rica. Rectoria. OPLAU. Plan Quinquenal de desarrollo de la Universidad de Costa Rica 1986-1990. Publicaciones de la Universidad de Costa Rica, 1985.

Universidad de Costa Rica. Vicerrectoria de Docencia. Resoluciones 1984-1985. 